\title{
THE INVERSE LIMIT OF THE FUNDAMENTAL GROUPS OF BRANCHED CYCLIC COVERINGS
}

\author{
MICHAEL DELLOMO
}

(Communicated by Haynes R. Miller)

\begin{abstract}
Cowsik and Swarup [CS] have shown that the homology groups of the infinite cyclic cover of a knot inject into the inverse limit of the homology groups of the branched cyclic covers. They also give conditions under which the injection is an isomorphism. We prove an analogous result for the fundamental group and generalize it to the case of links.
\end{abstract}

The relationship between the homology of the infinite cyclic cover of a knot complement and that of the inverse limit of the branched cyclic covers has been investigated in [CS, D1, D2]. We would like to extend some of these results to homotopy.

Let $K$ be a knot in $S^{3}, \tilde{X}$ the infinite cyclic cover of $X=S^{3}-K$, and $X_{n}$ the $n$-fold cyclic cover of $X$. We write $\Sigma_{n}$ for the branched $n$-fold cover of $S^{3}$ branched over $K$. This gives the following commutative diagram:

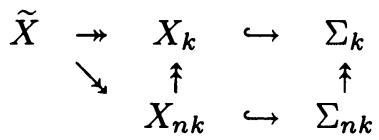

The sets $\left\{X_{k}\right\}$ and $\left\{\Sigma_{k}\right\}$ form inverse systems of topological spaces and we can construct the inverse limits $\hat{X}=\lim X_{k}$ and $\hat{\Sigma}=\lim \Sigma_{k}$ as well as inverse limits of their homology and homotopy groups. The diagram above induces maps $\widetilde{X} \rightarrow$ $\hat{X} \rightarrow \hat{\Sigma}$. Call the composition of these maps $i: \widetilde{X} \rightarrow \hat{\Sigma}$.

Cowsik and Swarup [CS] use commutative algebra to show that in homology we have the following.

THEOREM [CS]. The map $i_{*}: H_{*}(\tilde{X}) \rightarrow \lim H_{*}\left(\Sigma_{k}\right)=\check{H}_{*}(\hat{\Sigma})$ (Čech homology) is an injection.

They also give conditions under which $i_{*}$ is an isomorphism. $\check{H}_{1}(\hat{\Sigma})$ and the map $i_{*}$ are explicitly calculated in $[\mathbf{D 1}, \mathbf{D 2}]$.

What we would like to do now is investigate the map $i_{1}: \pi_{1}(\tilde{X}) \rightarrow \lim \pi_{1}\left(\Sigma_{k}\right)$. We have the following.

MAIN THEOREM. The map $i_{1}: \pi_{1}(\tilde{X}) \rightarrow \lim \pi_{1}\left(\Sigma_{k}\right)$ is injective. In fact, there $i s$ an exact sequence of the form $0 \rightarrow \pi_{1}(\widetilde{X}) \rightarrow \lim \pi\left(\Sigma_{k}\right) \rightarrow \lim ^{1} H_{k} \rightarrow \hat{Z} / Z \rightarrow 0$ where $H_{k}$ is the kernel of the map $\pi_{1}\left(X_{k}\right) \rightarrow \pi_{1}\left(\Sigma_{k}\right)$ and $\hat{Z}=\prod_{p} Z_{p}$, i.e., the product taken over all primes, $p$, of the p-adic integers, $Z_{p}$.

Received by the editors August 12, 1987 and, in revised form, October 14, 1987.

1980 Mathematics Subject Classification (1985 Revision). Primary 57M25, 55P55.

Research supported in part by SUNY Faculty Summer Research Fellowship. 
In order to obtain this result we must refer to the inverse limit (and $\lim ^{1}$ ) of a system of nonabelian groups. In this context we define the following (see [MS1]): Let $\left\{G_{k}\right\}$ be an inverse sequence of nonabelian groups with homomorphisms $p_{k, k+1}: G_{k+1} \rightarrow G_{k}$. Define

$$
\lim G_{k}=\left\{\text { sequences }\left(g_{k}\right) \in \prod_{k} G_{k} \mid p_{k, k+1}\left(g_{k+1}\right)=g_{k}\right\} .
$$

Define $\lim ^{1} G_{k}=\prod_{k} G_{k} / \sim$ where $\sim$ is defined as follows: for $x=\left(x_{k}\right), y=$ $\left(y_{k}\right) \subset \prod_{k} G_{k}$ we say $x \sim y$ provided there exists $\left(u_{k}\right) \in \prod_{k} G_{k}$ with $y_{k}=$ $u_{k} x_{k} p_{k, k+1}\left(u_{k+1}\right)$.

Note 1. If the groups are abelian, then the definitions reduce to the more familiar ones, for example, the map $d: \prod_{k} G_{k} \rightarrow \prod_{k} G_{k}$ defined by

$$
d\left(\left(x_{k}\right)\right)=\left(x_{k}-p_{k, k+1}\left(x_{k+1}\right)\right)
$$

has $\lim G_{k}$ as its kernel and $\lim ^{1} G_{k}$ as its cokernel.

Note 2. $\lim ^{1} G_{k}$ may not be a group if the $G_{k}$ are not abelian. Therefore we must be careful of how we interpret certain exact sequences. In particular, the following theorem is true for abelian and nonabelian groups:

THEOREM. Given inverse sequences $\left\{G_{k}\right\},\left\{G_{k}^{\prime}\right\}$, and $\left\{G_{k}^{\prime \prime}\right\}$ of groups and compatible exact sequences for each $k$, namely, commutative diagrams

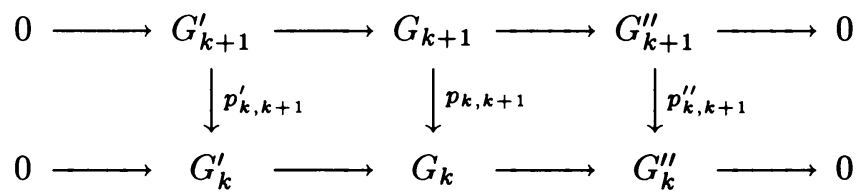

There is a natural exact sequence

$$
0 \rightarrow \lim G_{k}^{\prime} \rightarrow \lim G_{k} \rightarrow \lim G_{k}^{\prime \prime} \stackrel{\delta}{\rightarrow} \lim ^{1} G_{k} \rightarrow \lim ^{1} G_{k} \rightarrow \lim ^{1} G_{k}^{\prime \prime} \rightarrow 0
$$

It is understood that all maps to the right of and including $\delta$ are maps of sets. For the proof see [MS1].

Note 3. The above definitions are for inverse sequences. Since the systems we will be dealing with all have convenient cofinal subsequences, we will not need the more general version. A complete general treatment can be found in [BK, p. 307].

We now prove a lemma from which the Main Theorem will follow.

LEMMA. The sequence $0 \rightarrow \lim H_{k} \rightarrow \pi_{1}(\tilde{X}) \rightarrow \lim \pi_{1}\left(\Sigma_{k}\right) \rightarrow \lim ^{1} H_{k} \rightarrow$ $\hat{Z} / Z \rightarrow 0$ is exact.

PROOF OF THE LEMMA. Consider the system of exact sequences

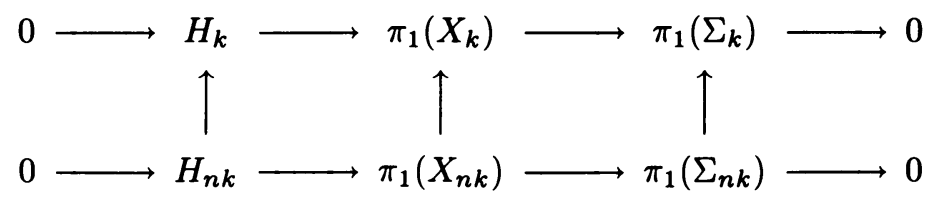


Passing to inverse limits, we get

$$
\begin{gathered}
0 \rightarrow \lim H_{k} \rightarrow \lim \pi_{1}\left(X_{k}\right) \rightarrow \lim \pi_{1}\left(\Sigma_{k}\right) \rightarrow \lim ^{1}\left(H_{k}\right) \\
\rightarrow \lim ^{1} \pi_{1}\left(X_{k}\right) \rightarrow \lim ^{1} \pi_{1}\left(\Sigma_{k}\right) \rightarrow 0 .
\end{gathered}
$$

Now we examine the individual terms of this exact sequence. Note that the $\lim ^{1}$ terms can be calculated by working with a cofinal subsequence, e.g., $\left\{H_{n !}\right\}$, $n=1,2, \ldots$.

First we look at $\lim ^{1} \pi_{1}\left(\Sigma_{k}\right)$. We can form the following sequence of sets $0 \rightarrow$ Ker $\rightarrow \pi_{0}(\hat{\Sigma}) \rightarrow \lim \pi_{0}\left(\Sigma_{k}\right) \rightarrow 0$. If the bonding maps of the inverse system were fibrations then this sequence would be exact with $\operatorname{Ker}=\lim ^{1} \pi_{1}\left(\Sigma_{k}\right)$. (See [MS1] for details.) Unfortunately they are not fibrations, but they do have a (nonunique) path lifting property. Therefore, we do get the above sequence, but the map Ker $\rightarrow \lim ^{1} \pi_{1}\left(\Sigma_{k}\right)$ is not necessarily one to one. It is, however, onto. (See [MS1, pp. 178-183]. Steps 1-3 of Lemma II.7.1.3 all work in this context.) Now $\pi_{0}(\hat{\Sigma})=0$ since $\hat{\Sigma}$ is path connected. Indeed there is a path from any point of $\hat{\Sigma}$ to the knot at its core. Therefore Ker $=0$ so $\lim ^{1} \pi_{1}\left(\Sigma_{k}\right)=0$.

REMARK. We could have shown this using a more geometric argument. The maps $\pi_{1}(\tilde{X}) \rightarrow \pi_{1}\left(\Sigma_{k}\right)$ can be shown to be surjective, hence the maps $\pi_{1}\left(\Sigma_{n k}\right) \rightarrow$ $\pi_{1}\left(\Sigma_{k}\right)$ are also surjective. We will use this argument later to determine what happens if $i_{1}$ is an isomorphism. For now, however, the lifting property argument allows us to point out the strong connectivity of $\hat{\Sigma}$, which is interesting in its own right.

Now consider $\lim \pi_{1}\left(X_{k}\right)$ and $\lim ^{1} \pi_{1}\left(X_{k}\right)$. Since $X_{n k} \rightarrow X_{k} \rightarrow X$ is a composition of covering maps, we have the following diagram:

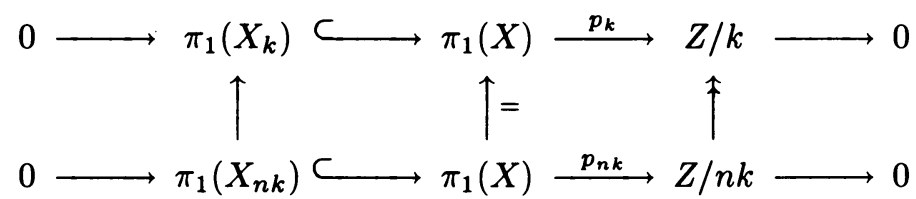

Passing to inverse limits we get

$$
0 \rightarrow \lim \pi_{1}\left(X_{k}\right) \rightarrow \pi_{1}(X) \stackrel{\hat{p}}{\rightarrow} \hat{Z} \rightarrow \lim ^{1} \pi_{1}\left(X_{k}\right) \rightarrow 0 .
$$

The maps $p_{k}$ factor as $\pi(X) \rightarrow Z \rightarrow Z / k$. So in the limit we have $\hat{p}: \pi(X) \rightarrow$ $Z \rightarrow \hat{Z}$. Now the kernel of $\pi_{1}(X) \rightarrow Z$ is $\pi_{1}(\tilde{X})$ so $\lim \pi_{1}\left(X_{k}\right) \cong \pi_{1}(\widetilde{X})$ and $\lim ^{1} \pi_{1}\left(X_{k}\right) \cong \hat{Z} / Z$.

The lemma now follows by substituting these results into exact sequence (1).

PROOF OF MAIN THEOREM. Let $e$ be the identity element of any group in question. It is enough to show that for each $g \in \pi_{1}(\widetilde{X}), g \neq e$, we can find a $k$ so that the image of $g$ in $\pi_{1}\left(\Sigma_{k}\right)$ is not the identity. It then follows that the image of $g \neq e$ in $\lim \pi_{1}\left(\Sigma_{k}\right)$. We require the following result due to Hempel [H].

THEOREM $[\mathbf{H}]$. The fundamental group of a Haken 3-manifold is residually finite, that is $\forall g \in \pi_{1}(X), g \neq e \exists$ a finite group $F$ and a homomorphism $h: \pi_{1}(X) \rightarrow$ $F$ with $h(g) \neq e \in F$.

Since knot complements are Haken, this theorem applies to our situation. 
Let $g \neq e$ be an element of $\pi_{1}(\tilde{X})$. We have $g \mapsto g^{\prime} \in \pi_{1}(X) . \pi_{1}(X)$ is residually finite so for some $k$ there exists a $k$ element group $F_{k}$ and a homomorphism $h$ with $h\left(g^{\prime}\right) \neq e \in F_{k}$. Let $\alpha$ be a small loop in $X$ that links once with the knot (i.e. a meridian of a regular solid torus neighborhood of $K$ ). Now if $\alpha=[\alpha] \in$ $\pi_{1}(X)$ then $a \mapsto 1 \in Z / k$ in the sequence $0 \rightarrow \pi_{1}\left(X_{k}\right) \rightarrow \pi_{1}(X) \rightarrow Z / k \rightarrow 0$. Therefore $a^{k} \in \pi_{1}\left(X_{k}\right)$. When we form $\Sigma_{k}$ from $X_{k}$ by sewing in a solid torus, all conjugates of the paths $\alpha^{k}$ become homotopically trivial. Specifically, we see that the normal subgroup generated by $a^{k}$ in $\pi_{1}\left(X_{k}\right)$ is precisely $H_{k}$ and that $\pi_{1}\left(\Sigma_{k}\right)$ can be described as the quotient of $\pi_{1}\left(X_{k}\right)$ by the relation $a^{k}=e$.

Let $N_{k}$ be the normal subgroup generated by $a^{k}$ in $\pi_{1}(X)$. This gives the following commutative diagram:

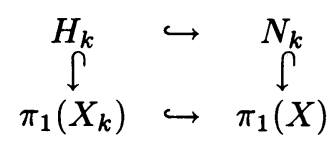

We can form the group $G=\pi_{1}(X) / N_{k}$. Since $h$ maps $\pi_{1}(X)$ to a finite group of order $k$ we have $\left.h\right|_{N_{k}}=0$, i.e., $h$ sends $N_{k}$ to the identity of $F_{k}$. Therefore $h$ induces a map $h^{\prime}: G \rightarrow F_{k}$ and a commutative triangle

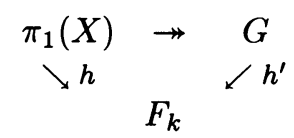

Combining the square and triangle above we have the commutative diagram

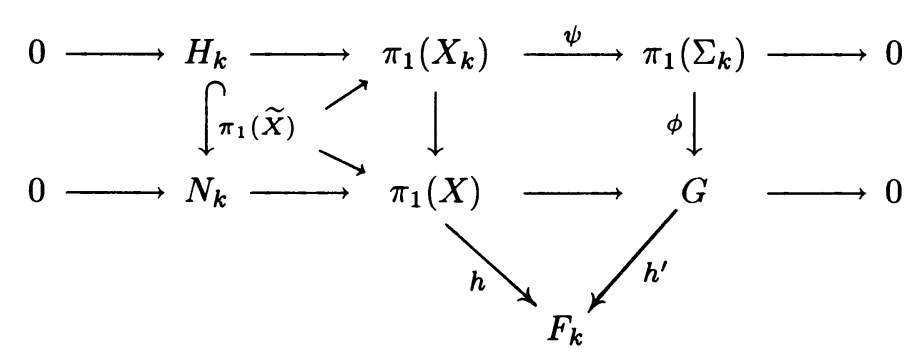

Now we return to $g \in \pi_{1}(\tilde{X})$. We have $g \mapsto g^{\prime} \in \pi_{1}(X)$ and $g \mapsto g_{k} \in \pi_{1}\left(X_{k}\right)$. The image of $g$ in $\pi_{1}\left(\Sigma_{k}\right)$ is $g_{k}^{\prime}=\psi\left(g_{k}\right)$. By commutativity, we have $h^{\prime}\left(\phi\left(g_{k}^{\prime}\right)\right) \neq e \in F_{k}$. Consequently $g_{k}^{\prime} \neq e \in \pi_{1}\left(\Sigma_{k}\right)$. This completes the proof of the Theorem.

Cowsik and Swarup go on to prove that the map $i_{*}$ is an isomorphism only when the Alexander polynomial of $K$ divides a cyclotomic polynomial. The analogous result for $i_{1}$ is considerably more restrictive:

THEOREM. The map $i_{1}: \pi_{1}(\tilde{X}) \rightarrow \lim \pi_{1}\left(\Sigma_{k}\right)$ is an isomorphism if and only if the knot, $K$, is trivial.

PROOF. If $K$ is trivial, the result is obvious since $\pi_{1}(\tilde{X})=\pi_{1}\left(\Sigma_{k}\right)=0$. So suppose $i_{1}$ is an isomorphism. Note that $\pi_{1}(\tilde{X}) \rightarrow \pi_{1}\left(\Sigma_{k}\right)$ is surjective for all $k$. Indeed, for any $x \in \pi_{1}\left(\Sigma_{k}\right)$, there is a loop $\alpha$ in $\Sigma_{k}$ with $[\alpha]=x$ which has zero linking number with the knot. $\alpha$ can also be chosen so that it does not intersect a small tubular neighborhood of $K$. Such an $\alpha$ will lift to a loop in $\tilde{X}$. We can 
therefore write down the following system of exact sequences

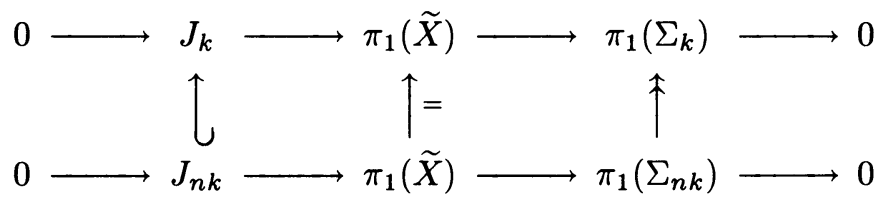

Passing to inverse limits, we have

$$
0 \rightarrow \lim J_{k} \rightarrow \pi_{1}(\tilde{X}) \rightarrow \lim \pi_{1}\left(\Sigma_{k}\right) \rightarrow \lim ^{1} J_{k} \rightarrow 0 .
$$

Now $i_{1}$ is an isomorphism so $\lim J_{k}=\lim ^{1} J_{k}=0$. Therefore the system $\left\{J_{k}\right\}$ is isomorphic to 0 (in the category of pro-groups; see [MS1, II.6.2, Theorem 12] for details). By the Morita lemma [MS1, DS] we have that $\forall n \exists m$ so that the following diagram commutes:

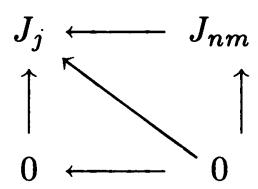

But since the map $J_{n m} \rightarrow J_{n}$ is an inclusion, we have that $J_{n m}=0$. Therefore $\exists L=n m$ with $\pi_{1}(\tilde{X}) \cong \pi_{1}\left(\Sigma_{L}\right)$.

Let $a$ be the homotopy class in $\pi_{1}(X)$ of a small loop around the knot. Then $\forall x \in \pi_{1}(\tilde{X})$ we have $a x a^{-1} \in \pi_{1}(\tilde{X})$. (This is the classical action of the meridian of $k$ on $\pi_{1}(\tilde{X})$.) Also we have $a^{L}=e \in \pi_{1}\left(\Sigma_{k}\right)$ so the isomorphism from $\pi_{1}(\tilde{X})$ to $\pi_{1}\left(\Sigma_{L}\right)$ sends $x$ and $a^{L} x a^{-L}$ to the same element. Therefore $a^{L}$ commutes in $\pi_{1}(X)$ with every element of $\pi_{1}(\tilde{X})=\left[\pi_{1}(X), \pi_{1}(X)\right]$. Furthermore, since $a$ maps to a generator of $\pi_{1}(X) / \pi_{1}(\widetilde{X}) \cong Z$ we have that $a^{L}$ commutes every element of $\pi_{1}(X)$. Therefore $a^{L}$ is in the center of $\pi_{1}(X)$.

Burde and Zieschang $[\mathbf{B Z}]$ showed that the only knots with nontrivial center are torus knots. For the $(p, q)$ torus knot with $p$ and $q$ relatively prime, the fundamental group of $X$ has the following presentation: $\pi_{1}(X)=\left\{x, y: x^{p}=y^{q}\right\}$ (see $[\mathbf{R}]$ ). The center of $\pi_{1}(X)$ is the cyclic group generated by $x^{p}$. With respect to this presentation, we can express one meridian as $x^{u} y^{v}$ where $v p+u q=1$. The other meridians can be written as conjugates of this one with respect to $x^{b}$.

We can assume that $a=x^{u} y^{v}$ (the proofs for the other choices are similar). If $K$ is nontrivial then $\left(x^{u} y^{v}\right)^{L}$ would have to be in the center and, hence, be equal to $x^{n p}$ for some $n$. But this is impossible since every element of $\pi_{1}(X)$ can be written uniquely in the form $x^{a_{1}} y^{b_{1}} \cdots x^{a_{m}} y^{b_{m}} w^{c}$ where $0 \leq a_{1}<p, 0<a_{i}<p \forall i>1$, $-q<b_{m} \leq 0,-q<b_{j}<0 \forall j>1$ and $w=x^{p}=y^{q}$ (see [MKS, Chapter 4] for details). This completes the proof of the theorem.

Finally we can use part of the proof of the Main Theorem to obtain an analogous result for links. In particular, let $X$ be the complement of an $l$ element link in $S^{3}$, let $\widetilde{X}$ be the universal abelian cover of $X$ and let $\left\{X_{A B}\right\}$ be the inverse system of the unbranched finite abelian covers of $X$. Write $\left\{\Sigma_{A B}\right\}$ for the inverse system of branched abelian covers of $S^{3}$ branched over $L$. We restrict our attention to the cofinal subsystems $\left\{X_{k}\right\}$ and $\left\{\Sigma_{k}\right\}$ where $\pi_{1}(X) / \pi_{1}\left(X_{k}\right) \cong \bigoplus_{i=1}^{l} Z / k$.

Since link complements are also Haken, we can apply the proof of the Main Theorem with only slight modifications. Choose meridians $a_{1}, \ldots, a_{l}$, one for each 
component of $L$ and construct the group $G$ by taking the quotient of $\pi_{1}(X)$ by the relations $a_{i}^{k}=e$. We eventually obtain a diagram similar to (2). Therefore we have COROLlaRY. For links, the map $i_{1}: \pi_{1}(\tilde{X}) \rightarrow \lim \pi_{1}\left(\Sigma_{A B}\right)$ is an injection.

\section{REFERENCES}

[BK] A. K. Bousfield and D. M. Kan, Homotopy limits, completions and localizations, Lecture Notes in Math., vol. 304, Springer-Verlag, Berlin and New York, 1972.

[BZ] G. Burde and H. Zieschang, Eine Kennzeichnung der Torus Knoten, Math. Ann. 167 (1966), 169-176.

[CS] R. C. Cowsik and G. A. Swarup, A remark on infinite cyclic covers, J. Pure Appl. Algebra 11 (1977), 131-138.

[D1] M. Dellomo, On the inverse limit of the finite branched cyclic covers of a knot, J. Pure Appl. Algebra 40 (1986), 15-26.

[D2] _ , Through the non-simply connected looking glass, or the inverse limit of finite branched covers, Ph.D. Thesis, Johns Hopkins Univ., 1984.

[DS] J. Dydak and J. Segal, Shape theory: An introduction, Lecture Notes in Math., vol. 688, Springer-Verlag, Berlin, 1978.

[H] J. Hempel, Residual finiteness for 3-manifolds, Ann. of Math. Studies (to appear).

[MKS] W. Magnus, A. Karras and D. Solitar, Combinatorial group theory, Dover, New York, 1976. [MS1] S. Mardesic and J. Segal, Shape theory, North-Holland, Amsterdam, New York, 1982.

[R] D. Rolfson, Knots and links, Publish or Perish, Berkeley, Calif., 1976.

Department of Mathematics, State University of NeW York at BinghamTON, BINGHAMTON, NEW YORK 13901 $\mathrm{Z} 431$

Current address: Mitre Corporation, 1820 Dolley Madison Boulevard, McLean, Virginia 22102 